\title{
A boundary-value problem arising in the modelling of equatorial wind-drift currents
}

\author{
Kateryna Marynets ${ }^{1}[0$
}

Received: 26 January 2021 / Accepted: 26 March 2021 / Published online: 7 April 2021

(c) The Author(s) 2021

\begin{abstract}
We investigate the boundary-value problem that models wind-induced equatorial flows, establishing the existence and uniqueness of solutions. We also discuss some special cases that were studied in recent geophysical research.
\end{abstract}

Keywords Boundary-value problem · Second order equations · Geophysical flows

Mathematics Subject Classification 35Q35 · 34B05 · 76U60

\section{Introduction}

The transfer of energy from the wind to the ocean is a fundamental aspect of geophysical research studies. At mid-latitudes and in polar regions the main mechanism is explained by means of Ekman theory (see [18]), whose classical aspects were shown recently (see the discussions in $[2,3,12]$ ) to be in need of further adjustment to bring the theoretical predictions closer to what field data reveals. In equatorial oceanic regions, it is well-established that Ekman theory fails (see the discussion in [1,8]). In particular, there is no deflection of the surface current with respect to the wind, since at the Equator the peculiar nature of the Coriolis force imposes an azimuthal direction of propagation. This appears to be a simplification, but in reality the nature of equatorial currents is quite intricate and of great current interest. One recent approach that proved to be very successful consists to analyse the problem as a large-scale flow in rotating spherical coordinates (see [6,7,14-16]). The fine details are however difficult to extract from this type of investigation, and an alternative approach was purused

Communicated by Adrian Constantin.

Kateryna Marynets

K.Marynets@tudelft.nl

1 Kateryna Marynets

Faculty of Electrical Engineering, Mathematics and Computer Science, Delft Institute of Applied Mathematics, Delft University of Technology, Mekelweg 4, 2628 CD Delft, The Netherlands 
in $[5,13])$. The latter approach simplifies the geometry by relying on the equatorial $f$-plane approximation, but compensates this by a higher precision with respect to a two-layer stratification and the distribution of the eddy viscosity. One advantage is that the core of the flow, consisting of the azimuthal velocity component throughout the depth, can be made available in explicit analytic form, and thus specific properties can be studied in detail without recourse to numerical simulations-these are only needed when studying perturbations of this background flow. In particular, the depth of the thermocline is known to be an important dynamical factor, as shown by the behaviour of the flow during an El Nino year in the Pacific, when the thermocline is much nearer the surface than usually (for more details about the El Nino effect we refer to [17]). These comments motivate us to present an approach that is applicable for the background equatorial flow for an arbitrary (stable) stratification and an arbitrary distribution of the vertical eddy viscosity. Consequently, in this paper we present an analytic approach that provides a generally-valid explicit formula for the azimuthal velocity component, obtained as the unique solution to the boundary-value problem that models equatorial wind-drift currents. Due to its importance for the global climate, we pursue an in-depth study pertaining to the setting of the Pacific Ocean, but our considerations can be adapted to consider equatorial flows in the Atlantic Ocean or in the Indian Ocean. For the Atlantic Ocean the changes are only quantitative, so that our approach applies actually directly, but in the Indian Ocean the onset of the monsoon season leads to changes in the direction of the prevailing winds, so that one has to be careful in keeping or reversing the azimuthal orientation of the sub-surface azimuthal flow. Concerning the relation of our continuous stratification setting to a two-layer stratified fluid, since we work directly with the governing equations, the latter can be recovered by a limiting process in our main integral formula for the azimuthal velocity field, thus bypassing the need to consider weak formulations of the governing equations.

\section{Main result}

Consider a calm setting, disturbed by a sudden westward wind-stress that acts on the surface of the ocean, practically unaltered for a long time-a situation that is typical for the trade winds in the equatorial region of the Pacific Ocean (as mentioned in the Introduction, this is also the case for the Atlantic Ocean but in the Indian ocean one has to adapt the context to the relevant monsoon season). In the open sea, with a constant ocean depth and with a uniform wind stress acting on the ocean surface, no gradient of sea-surface elevation will occur [11] but the initially (constant) atmospheric pressure at the ocean surface changes and a subsurface current field is generated in the ocean. If the wind ceases to act-this is the case, for example, with the onset of the El Nino phenomenon-then the pressure at the surface is rapidly adjusting to the (constant) atmospheric pressure but the underlying current will persist for some considerable time (see the data in [5]). Indeed, the qualitative flow pattern in the equatorial Pacific ocean remains in place, even if quantitative changes which affect the global climate take place (for example, the heavy rainfall in Peru associated with the occurrence of an El Nino event). These quantitative changes are captured 
by the depth of the thermocline and by changes in the density (these being related to atmospheric temperature variations) and in the eddy viscosity (which is directly connected to the strength of the wind bursts). This background flow typically interacts with waves (see the study [4]) but in this paper we will concentrate solely on the features of the ocean currents. Also, with respect to the recent approaches in $[15,16]$, where we relied on extending the spherical-geometry approach in [8], we adopt now the alternative setting of the equatorial $f$-plane approximation. The more local nature of the present approach (which contrasts to the global features ensured by studies performed in rotating spherical coordinates, as highlighted in [7]) is compensated by the flexibility that it offers with respect to the above-mentioned factors that influence the quantitative aspects of the flow pattern.

We choose a coordinate system with the origin at a point along the Equator on the Earth's surface, with the $\bar{x}$-axis chosen horizontally due East and the $\bar{z}$-axis upward; generally we denote physical quantities with an overbar. Under the assumption of a uniform wind stress, we consider the linearised Navier-Stokes and mass conservation equations for a forced steady-state flow, with a vanishing vertical-fluid-velocity component in the $f$-plane approximation in the region $-\bar{d}<\bar{z}<0$ delimited by the flat free surface $\bar{z}=0$ and by the flat, impermeable ocean bed $\bar{z}=-\bar{d}$ (with $\bar{d}$ about 4 $\mathrm{km})$ :

$$
\begin{cases}0 & =-\frac{1}{\bar{\rho}} \bar{P}_{\bar{x}}+\left(\bar{v} \bar{u}_{\bar{z}}\right)_{\bar{z}}, \\ -2 \bar{\Omega} \bar{u} & =-\frac{1}{\bar{\rho}} \bar{P}_{\bar{z}}-\bar{g}, \\ \bar{u}_{\bar{x}} & =0,\end{cases}
$$

where $\bar{u}$ is the horizontal fluid velocity component (with vanishing meridional and vertical ones), $\bar{\Omega}$ is the (constant) rotational speed of the Earth round the polar axis toward the East, having the approximate magnitude $7.29 \times 10^{-5} \mathrm{rad} \mathrm{s}^{-1}, \bar{g} \approx 9.8$ $\mathrm{m} \mathrm{s}^{-1}$ is the (constant) gravitational acceleration at the Earth's surface, $\bar{\rho}(\bar{z})$ is the water's density, $\bar{P}$ is the pressure, and $\bar{v}(\bar{z})$ is the vertical eddy viscosity parameter (its depth-dependence being consistent with measurements — see the discussion in [10]). The boundary conditions associated with the system (1) are

$$
\bar{u}=0 \text { on } \bar{z}=-\bar{d}
$$

(expressing the fact that the wind effects negligible in abyssal ocean regions),

$$
\bar{u}_{\bar{z}}=0 \text { on } \bar{z}=-\bar{h}
$$

where $\bar{z}=-\bar{h}$ is the thermocline (expressing the fact that the shear-and thus the stress - vanishes at the thermocline, as is realistic for the central Pacific region, according to the discussion in [10]), and

$$
\bar{u}_{\bar{z}}=-\bar{\alpha} \text { on } \bar{z}=0
$$


(expressing the knowledge of the surface wind shear), where $\bar{\alpha}$ is a (dimensional) constant. For the physical motivation of the boundary condition (4) we refer to the detailed discussion in [5], but we point out the fact that (4) ensures that shear at the surface does not depend on the wind speed. This feature is essential in our considerations, since it allows us to proceed with the study of the wind-drift ocean currents without having to pay special attention to wind bursts. Let us also note that the sign of the right-hand side in (4) is motivated by the fact that in the equatorial Pacific the trade winds blow westwards.

We differentiate the outcome of the first equation in (1) after multiplication by $\bar{\rho}$ with respect to the $\bar{z}$-variable and use the outcome of differentiating the second equation in (1) with respect to the $\bar{x}$-variable to infer that

$$
\bar{\rho}\left(\bar{v} \bar{u}_{\bar{z}}\right)_{\bar{z}}=-\bar{\beta}, \quad-\bar{d}<\bar{z}<0
$$

for some dimensional constant $\bar{\beta}$, whose sign we anticipated. Taking now (3) into account, we get

$$
\bar{v}(\bar{z}) \bar{u}_{\bar{z}}(\bar{z})=-\bar{\beta} \int_{-\bar{h}}^{\bar{z}} \frac{1}{\bar{\rho}(\bar{s})} \mathrm{d} \bar{s}, \quad-\bar{d} \leq \bar{z} \leq 0 .
$$

The boundary condition (4) now determines the dimensional constant

$$
\bar{\beta}=\frac{\bar{\alpha} \bar{\nu}(0)}{\int_{-\bar{h}}^{0} \frac{1}{\bar{\rho}(\bar{s})} \mathrm{d} \bar{s}}
$$

so that (5) yields

$$
\bar{u}_{\bar{z}}(\bar{z})=-\frac{\bar{\alpha} \bar{\nu}(0)}{\bar{\nu}(\bar{z})} \frac{\int_{-\bar{h}}^{\bar{z}} \frac{1}{\bar{\rho}(\bar{s})} \mathrm{d} \bar{s}}{\int_{-\bar{h}}^{0} \frac{1}{\bar{\rho}(\bar{s})} \mathrm{d} \bar{s}}, \quad-\bar{d} \leq \bar{z} \leq 0 .
$$

The fact that the wind effects are confined to a near-surface layer, expressed by (2), now provides us with an explicit formula for the wind-drift current (Fig. 1):

$$
\bar{u}(\bar{z})=-\bar{\alpha} \bar{v}(0) \int_{-\bar{d}}^{\bar{z}} \frac{1}{\bar{\nu}(\bar{s})} \frac{\int_{-\bar{h}}^{\bar{s}} \frac{1}{\bar{\rho}(\bar{r})} \mathrm{d} \bar{r}}{\int_{-\bar{h}}^{0} \frac{1}{\bar{\rho}(\bar{r})} \mathrm{d} \bar{r}} \mathrm{~d} \bar{s}, \quad-\bar{d} \leq \bar{z} \leq 0 .
$$

From (7) and (8) we can draw a number of qualitative features of the wind-drift current flow:

- the current speed increases with height above the bed, from zero at the flat bed to its maximal value at the thermocline, and then it decreases, being westward at the surface (as it should, because the trade winds blow westwards in the equatorial Pacific); 




Fig. 1 The solution (8) captures all the main qualitative features of the Equatorial Undercurrent: flowreversal from a westward surface current to an eastward undercurrent that reaches maximal speed at the thermocline, and slow abyssal motion

- the overall shape of the current matches that obtained in [6] within an inviscid setting of rotating spherical coordinates, but here we can trace the exact source of the monotonicity changes since the quantitative details depend on the depth of the thermocline, on the size of the vertical eddy diffusivity and on that of the surface wind shear;

- as one can easily infer from (7), the flow in the ocean presents non-zero vorticity, so that the wave-current interactions are technically more challenging than those of irrotational flows (see the discussions in [4] and [9]).

\section{Some special cases of the general result}

The approach presented in the previous section investigates a more general setting than that pursued in the publications [5] and [13], where a two-layer density distribution was considered (in a stable stratification, with a constant density above the thermocline and a slighltly larger constant density below the thermocline, in the abyssal region), that is

$$
\bar{\rho}(\bar{z})= \begin{cases}\bar{\rho}_{0}, & -\bar{h}<\bar{z} \leq 0, \\ (1+r) \bar{\rho}_{0}, & -\bar{d} \leq \bar{z}<-\bar{h},\end{cases}
$$

with $r \approx 5 \times 10^{-3}$. The general conclusions apply, but the expression for the solution is tractable, especially if, in addition, we follow [10], assuming that turbulence measurements indicate that eddy viscosity decays exponentially with depth. On the other hand, as in the considerations made recently in [12] for Ekman flows (typical for oceanic regions away from the Equator), we can also impose that the eddy viscosity 
is piecewise linear, with a jump across the thermocline, so that

$$
\bar{v}(\bar{z})= \begin{cases}\bar{v}_{0}, & -\bar{h}<\bar{z} \leq 0, \\ \bar{v}_{1}, & -\bar{d} \leq \bar{z}<-\bar{h},\end{cases}
$$

where the (dimensional) constants satisfy $\bar{v}_{1}<\bar{v}_{0}$, given that deep down, in the abyssal layer beneath the thermocline, the effect of the wind is definitely less significant than in the near-surface layer.

The results presented in the previous section provide the main character of a purely azimuthal flow, that is typically perturbed, giving rise to non-zero meridional and vertical flow velocity components and to waves. In particular, waves could arise as oscillations of the free surface (surface gravity waves) but, more importantly from the point of view of energy transport, as perturbations of the thermocline (internal waves). Analytic investigations regarding two-dimensional waves that propagate in the azimuthal direction were initiated in [4,5], under simplifying assumptions about the vorticity - the piecewise constant case. To deal with the more general background state described in the present paper, it appears that a numerical approach is advisable.

Open Access This article is licensed under a Creative Commons Attribution 4.0 International License, which permits use, sharing, adaptation, distribution and reproduction in any medium or format, as long as you give appropriate credit to the original author(s) and the source, provide a link to the Creative Commons licence, and indicate if changes were made. The images or other third party material in this article are included in the article's Creative Commons licence, unless indicated otherwise in a credit line to the material. If material is not included in the article's Creative Commons licence and your intended use is not permitted by statutory regulation or exceeds the permitted use, you will need to obtain permission directly from the copyright holder. To view a copy of this licence, visit http://creativecommons.org/licenses/by/4.0/.

\section{References}

1. Boyd, J.P.: Dynamics of the Equatorial Ocean. Springer, Berlin (2018)

2. Bressan, A., Constantin, A.: The deflection angle of surface ocean currents from the wind direction. J. Geophys. Res. Oceans 124, 7412-7420 (2019)

3. Constantin, A.: Frictional effects in wind-driven ocean currents. Geophys. Astrophys. Fluid Dyn. (2020). https://doi.org/10.1080/03091929.2020.1748614

4. Constantin, A., Ivanov, R.I.: Equatorial wave-current interactions. Commun. Math. Phys. 370, 1-48 (2019)

5. Constantin, A., Johnson, R.S.: The dynamics of waves interacting with the equatorial undercurrent. Geophys. Astrophys. Fluid Dyn. 109, 311-358 (2015)

6. Constantin, A., Johnson, R.S.: An exact, steady, purely azimuthal equatorial flow with a free surface. J. Phys. Oceanogr. 46, 1935-1945 (2016)

7. Constantin, A., Johnson, R.S.: Steady large-scale ocean flows in spherical coordinates. Oceanography 31, 42-50 (2018)

8. Constantin, A., Johnson, R.S.: Ekman-type solutions for shallow-water flows on a rotating sphere: a new perspective on a classical problem. Phys. Fluids 31, 021401 (2019)

9. Constantin, A., Strauss, W., Varvaruca, E.: Global bifurcation of steady gravity water waves with critical layers. Acta Math. 217, 195-262 (2016)

10. Cronin, M.F., Kessler, W.S.: Near-surface shear flow in the tropical Pacific cold tongue front. J. Phys. Oceanogr. 39, 1200-1215 (2009)

11. Davies, A.M.: Modelling storm surge current structure. In: Dyke, P.P.G., Moscardini, A.O., Robson E.H. (eds.) Offshore and Coastal Modelling, pp. 55-81. Wiley, New York (2013) 
12. Dritschel, D.G., Paldor, N., Constantin, A.: The Ekman spiral for piecewise-uniform viscosity. Ocean Sci. 16, 1089-1093 (2020)

13. Martin, C.I.: Dynamics of the thermocline in the equatorial region of the Pacific ocean. J. Nonlinear Math. Phys. 22, 516-522 (2015)

14. Martin, C.I.: On the existence of free-surface azimuthal equatorial flows. Appl. Anal. 96, 1207-1214 (2017)

15. Marynets, K.: A hyperbolic-type azimuthal velocity model for equatorial currents. Appl. Anal. (2020). https://doi.org/10.1080/00036811.2020.1774054

16. Marynets, K.: The modeling of the Equatorial Undercurrent using the Navier-Stokes equations in rotating spherical coordinates. Appl. Anal. https://doi.org/10.1080/00036811.2019.1673375

17. Talley, L.D., Pickard, G.L., Emery, W.J., Swift, J.H.: Descriptive Physical Oceanography: An Introduction. Elsevier, London (2011)

18. Vallis, G.K.: Atmosphere and Ocean Fluid Dynamics. Cambridge University Press, Cambridge (2016)

Publisher's Note Springer Nature remains neutral with regard to jurisdictional claims in published maps and institutional affiliations. 